\title{
La ética en los procesos de selección para la ejecución de obras públicas
}

\section{Ethics in the selection processes for the execution of public works}

\author{
Gloria M. Delgado Vera $\square$ \\ Universidad Nacional Mayor de San Marcos. Lima, Perú.
}

Recibido: 20-12-2018 Revisado: 19-04-2019 Aceptado: 11-05-2019 Publicado: 25 - 05-2019

\section{RESUMEN}

Actualmente, debido a diversos casos de corrupción, diversos espacios académicos y no académicos se han concentrado en evaluar y comprender cómo se desarrollan los procesos de contratación de obras públicas.

Debido a los descubrimientos de estudios periodísticos, en diversos países, especialmente en la región, se han hallado casos de corrupción en funcionarios de todo nivel. Ante esta situación, una evaluación ética resulta relevante e importante.

El presente estudio busca comprender, desde una vertiente de gestión y alta dirección, desde la sociología y la neurociencia, el porqué del inadecuado proceder de los funcionarios públicos anteriormente mencionados.

Esto en un marco de afirmación que indica que, si no nos encargamos de evaluar aspectos éticos y humanísticos, no podremos evidenciar un desarrollo sostenible en espacios públicos y privados.

Palabras clave: Bioética, corrupción, obras públicas.

\begin{abstract}
Currently, due to various cases of corruption, various academic and non-academic spaces have focused on evaluating and understanding how public works contracting processes are developed.

Due to the discoveries of journalistic studies, in several countries, especially in the region, cases of corruption have been found in officials of all levels. Given this situation, an ethical evaluation is relevant and important.

The present study seeks to understand, from a management and top management perspective, from sociology and neuroscience, the reason for the inappropriate behavior of the aforementioned public officials.

This in a frame of affirmation that indicates that, if we do not take care of evaluating ethical and humanistic aspects, we will not be able to have a sustainable development in public and private spaces.
\end{abstract}

Keywords: Bioethics, corruption, public works. 


\section{INTRODUCCIÓN}

El Perú en las últimas tres décadas inicio un proceso de crecimiento positivo que ha permitido la realización de importantes obras públicas que favorecen el desarrollo interno de la nación.

Esto de acuerdo a los datos encontrados en la publicación del Centro de Estudios de Responsabilidad Social, Emprendimiento y Sosteniblidad - CERES, de la Pontificia Universidad Católica del Perú (CENTRUM, 2017).

En este sentido, el crecimiento económico está ligado a la inversión pública y por ende a la formulación de proyectos para la ejecución de obras públicas.

Por lo tanto, para llevar a cabo dichas obras primero deben realizarse una serie de procedimientos, que requieren de la participación de personal especializado y calificado en la materia, quienes basados en la Ley de Contrataciones del Estado y su reglamento analizan, evalúan, califican y finalmente seleccionan al contratista que reúne los requisitos necesarios para la ejecución de la obra.

El proceso de selección se basa en los siguientes pasos: la convocatoria pública, el registro de los participantes, formulación y absolución de consultas, formulación y absolución de observaciones, integración de las bases, presentación de las propuestas, calificación y evaluación de las propuestas y finalmente el otorgamiento de la buena pro.

Como en todo acto administrativo, se presume que la actuación de los funcionarios responsables de la evaluación de expedientes para la adjudicación de la
Buena Pro se basa en los principios de transparencia y ética que debe regir la actuación de todo funcionario público.

A pesar de ello, se observan con frecuencia casos en los cuales el proceso se ha visto viciado, debido al ofrecimiento y pago de altas sumas de dinero por parte de algunos contratistas los cuales tienen por finalidad salir favorecidos en dicho proceso.

El presente trabajo se ocupa de la descripción de los procesos administrativos a seguirse para la ejecución de obras públicas y la actuación ética que deben guardar los funcionarios encargados de cumplir con estas funciones, ya que para nadie es un secreto que en esta área se han presentado sonados casos de corrupción, entre los que se podría mencionar el caso Lava Jato, tan solo por tocar uno de los más emblemáticos y conocidos.

En este sentido, el Estado a través del Organismo Superior de Contrataciones del Estado (OSCE) ha asumido el compromiso de crear mecanismos más efectivos para contrarrestar esta realidad.

\section{SITUACIÓN PROBLEMÁTICA}

Hasta finales de la década de los noventa, el Perú no contaba con una normativa legal que regulara y estableciera reglas claras en cuanto a los procesos de selección a contratistas para la elaboración de obras públicas, adquisición de bienes o de servicios.

En ese entonces, los criterios se regían dependiendo específicamente de cada 
caso o atendiendo a necesidades contextualizadas.

Esta realidad cambió cuando: "En el año 1998 se crea la Ley 26850, Ley de Contrataciones y Adquisiciones del Estado, iniciándose el proceso de unificación de la normativa y de los regímenes coexistentes hasta entonces, creándose el Consejo Superior de Contrataciones y Adquisiciones del Estado - CONSUCODE (hoy OSCE)". (Hernández, 2012).

En la actualidad, cuando se inicia un proceso de selección para la contratación de obras públicas, deben seguirse los pasos contemplados en la Ley de Contrataciones del Estado y su Reglamento.

Sin embargo, durante las etapas del proceso de contratación, los ganadores de estos concursos obtienen el contrato a través de vías no legales, siendo en el mayor de los casos el soborno a alguno o todos los funcionarios que conforman los comités de selección o a sus superiores.

Los estudios sobre el tema señalan que la contratación pública resulta:

Conflictiva y poco eficaz, y con frecuencia causa daño patrimonial al Estado por dos vías: la primera, por las demandas que pierde el Estado debido a la mala gestión de los contratos y, la segunda, por la corrupción imperante en los procesos de selección En ambos casos la discrecionalidad de los funcionarios públicos tiene una alta incidencia en el daño que se causa al Estado. (Gorbaneff y Cabarcas, 2009)

Sobre el particular, la mayoría de los estudios reseñan que la gestión de la contratación pública debe regirse por principios orientadores formales como la igualdad, libre concurrencia, seguridad jurídica, entre otros.

Sin embargo, también, se plantean otros principios que son estrictamente éticos o de probidad y que son exigibles a todo funcionario público, e incluso a los privados cuando se relacionan con la administración pública (Campos, 2007).

Todo esto constituye el universo de lo que debe suceder o del cómo se deben asumir los contratos con las entidades que prestan servicios u ofrecen la ejecución de obras para el estado. Sin embargo, vemos cómo la corrupción arropa este sector.

Los funcionarios obviando los principios fundamentales de la ética y pasando por sobre las normativas establecidas legalmente reciben diferentes beneficios personales.

Respecto a este particular, algunos estudios han intentado dilucidar las causas que inducen a un individuo a cometer actos de corrupción.

Una primera perspectiva planteada por Rose Ackerman (2010) señala que las causas podrían encontrarse directamente en las necesidades económicas de los empleados y funcionarios, ya que muchos de ellos poseen salarios insuficientes que no cubren todas sus necesidades básicas, así como la convivencia en un ambiente laboral que no ofrece la motivación apropiada para cumplir sus labores.

Sin embargo, esta realidad por sí sola no constituye un motivo valedero para justificar ni avalar la corrupción. 
Por su parte, (Zegarra, 2002) considera y estudia la corrupción y la falta de ética de los funcionarios como un fenómeno digno de ser analizado por parte de la ciencia política en búsqueda de las causas directas que llevan al ser humano a cometer este tipo de actos.

Otro autor como es el caso de (Mujica, 2011) señala directamente que la corrupción y la falta de ética de los funcionarios públicos en el Perú debe ser estudiada y visualizada directamente como un fenómeno "cultural".

Con lo revisado hasta ahora, podemos señalar que los autores consultados exponen sus puntos de vista acerca de las diferentes causas de la corrupción, la misma que puede darse a través de las famosas coimas, dádivas, regalos, favores políticos, sobornos e incluso extorsiones.

Esto se ejecuta por medio de acuerdos y pactos entre las partes representantes del sector público y privado, quienes en búsqueda de beneficios económicos fraguan planes que implican actos ilícitos.

De acuerdo con Campos: "La corrupción se encuentra presente en las compras estadales debido al manejo de grandes sumas de dinero" (2007). Otra investigación relevante es la de Peña (2005), quien interpreta los actos de corrupción como un producto de la ambición personal.

Sin embargo, a primera vista, este criterio podría parecer muy simplista y pudiéramos decir que la corrupción y la falta de ética para el manejo del dinero público implica además de todo lo dicho hasta ahora por parte de los autores citados, un proceso en el cual se interrelacionan los individuos, sin menoscabar el componente que por supuesto motiva la ambición.
Llegado a este punto del trabajo, es necesario definir claramente a qué nos referimos con el término corrupción, pues hasta ahora solo hemos abordado los preceptos que tratan de describirla y explicarla. Morales (2009) define la corrupción como: "aquel acto en el que se produce un "abuso de la función pública para beneficios privados" o particulares o para obtener ganancias personales".

Esta definición nos obliga a plantearnos la corrupción desde dos puntos de vista, como acto económico y como un fenómeno social; el primero con las implicancias perjudiciales que esta conlleva para el modelo de Estado, y la segunda, cómo se percibe dependiendo de la sociedad en la que se hayan cometido los hechos, lo que en un país puede ser considerado como corrupción quizá en otro no lo sea o no tenga implicancias tan graves o los hechos sean sencillamente inadmisibles.

Ante este panorama, es interesante inclusive tratar de dilucidar ¿Cómo funciona la mente de un corrupto? Pues para nuestra sorpresa, durante la investigación para llevar a cabo el presente ensayo, hemos descubierto que existe un mecanismo neurobiológico que puedeexplicarlafalta deética o deshonestidad. Según un estudio del University College de Londres, publicado en Nature Neuroscience, el cerebro humano es capaz de aceptar y adaptarse a la deshonestidad (Huerta, 2016).

Los estudios demostraron que con los primeros actos deshonestos la amígdala cerebral se activaba fuertemente, pero con cada subsecuente acto deshonesto su actividad disminuía progresivamente; es decir, la amígdala cerebral se iba 'acostumbrando' a los actos deshonestos.

En resumen, al perder la actividad de su amígdala cerebral, la persona va perdiendo 
el miedo y se acostumbra al delito. En consecuencia, el corrupto avezado va aumentando la magnitud de sus actos deshonestos.

El gran corrupto pierde completamente la actividad de su amígdala cerebral. En resumen, la biología y la sociología han tratado de buscar explicación al por qué se dan tantos actos de corrupción en los entornos donde se maneja el dinero.

Ahora bien, los tipos de actos de corrupción aparentemente están ligados a sus causas. La mayoría de las investigaciones han girado sobre dos grandes ejes: las implicancias económicas de la corrupción y la mirada jurídica sobre ella.

En relación al ámbito económico, algunos estudios han tratado de medir los niveles de corrupción y ver sus implicancias (Morales, 2009), mientras otros tienen como interés diseñar sistemas de control que permitan atenuar la corrupción (Artavia, 2008).

Si bien es cierto los trabajos revisados plantean diversos conceptos respecto de la definición de corrupción, parece existir un acuerdo entre diversos autores que indican que el cargo público y la corrupción están íntimamente vinculados en la práctica (Mujica, 2011).

Corresponde en este punto de la investigación explicar en qué etapa de los procesos de selección para la ejecución de obras públicas se llevan a cabo los diferentes hechos de corrupción y sus respectivas implicancias legales, políticas y económicas.

En el Perú, como ya mencionamos con anterioridad, existe una normativa legal que rige los procesos de contrataciones.
Se trata de la Ley de contrataciones del estado, la cual data de la década de los noventa, pero que a lo largo del tiempo ha venido sufriendo una serie de transformaciones, las cuales no siempre han favorecido ni agilizado el proceso, sino que por el contrario han generado nuevas restricciones para la participación de otros contratistas y al parecer favorecido a grandes grupos de poder.

La última modificación de la ley de Contrataciones del Estado y su Reglamento se realizó en el mes de septiembre del presente año, cuando a través del decreto legislativo $N^{\circ}$ 1444, promulgado con la finalidad de impulsar la ejecución de políticas públicas nacionales y sectoriales mediante la agilización de los procesos de contratación; así como fortalecer al Organismo Supervisor de las Contrataciones del Estado (OSCE) y a la Central de Compras Públicas - Perú Compras para fomentar la eficiencia en las contrataciones.

El Artículo 2 incluye la modificación de diversos artículos de la Ley $\mathrm{N}^{\circ} 30225$, estos son el 3, 4, 5, 6, 7, 8, 10, 11, 13, 14, 15, 16, 17, $18,19,22,23,24,26,27,28,29,31,33,34$, $38,40,41,44,45,49,50,52,58,59$ y Primera Disposición Complementaria Final de la Ley N³0225, Ley de Contrataciones del Estado.

En la mayoría de los casos, las Instituciones Públicas conforman comités de selección con la finalidad de que se encarguen de realizar todo el seguimiento, monitoreo, calificación y evaluación del Proceso de Selección.

Este procedimiento se contempla en el artículo 6: 8.2 La Entidad puede conformar comités de selección, los cuales son órganos colegiados y autónomos, encargados de seleccionar al proveedor que brinde los bienes, servicios u obras requeridos por 
el área usuaria a través de determinada contratación. El reglamento establece su composición, funciones, responsabilidades, entre otros. 8.3 El Titular de la Entidad puede delegar, mediante resolución, la autoridad que la presente norma le otorga.

Puede delegar, al siguiente nivel de decisión, las autorizaciones de prestaciones adicionales de obra.

La declaración de nulidad de oficio y la aprobación de las contrataciones directas no pueden ser objeto de delegación, salvo lo dispuesto en el reglamento. 8.4 El reglamento establece otros supuestos en los que el Titular de la Entidad no puede delegar la autoridad otorgada".

Esta misma Ley comprende los mecanismos para evitar actos de corrupción y delitos.

El mismo se explica en su artículo 14.1 Cuando la Entidad, el Organismo Supervisor de las Contrataciones del Estado (OSCE) - el Tribunal de Contrataciones del Estado verifique la existencia de indicios de conductas anticompetitivas en un procedimiento de selección en los términos de la normativa especial, debe remitir toda la información pertinente a la Secretaría Técnica de la Comisión de Defensa de la Libre Competencia del INDECOPI para que ésta, de ser el caso, inicie el procedimiento administrativo sancionador correspondiente contra los presuntos responsables.

Esta decisión debe ser mantenida en reserva y no debe ser notificada a los presuntos responsables a fin de asegurar la eficacia de la investigación.

Para que el comité de selección pueda realizar todo el proceso para la adjudicación de la Buena Pro de una obra, esta debe estar contemplada en el plan anual de contrataciones.

Al respecto, el artículo 15 establece: Formulación del Plan Anual de Contrataciones: Teniendo en cuenta la etapa de formulación y programación presupuestal correspondiente al siguiente año fiscal, cada Entidad debe programar en el cuadro de necesidades los requerimientos de bienes, servicios y obras necesarios para el cumplimiento de sus objetivos y actividades para dicho año, los que deben encontrarse vinculados al Plan Operativo Institucional, con la finalidad de elaborar el Plan Anual de Contrataciones.

15.2 El Plan Anual de Contrataciones que se apruebe debe prever las contrataciones de bienes, servicios y obras cubiertas con el Presupuesto Institucional de Apertura, con independencia de que se sujeten al ámbito de aplicación de la presente Ley o no, y de la fuente de financiamiento.

15.3 El Plan Anual de Contrataciones se publica en el Sistema Electrónico de Contrataciones del Estado (SEACE) y en el portal institucional de la respectiva Entidad.

En la figura 1 se detalla el proceso para la contratación pública: 


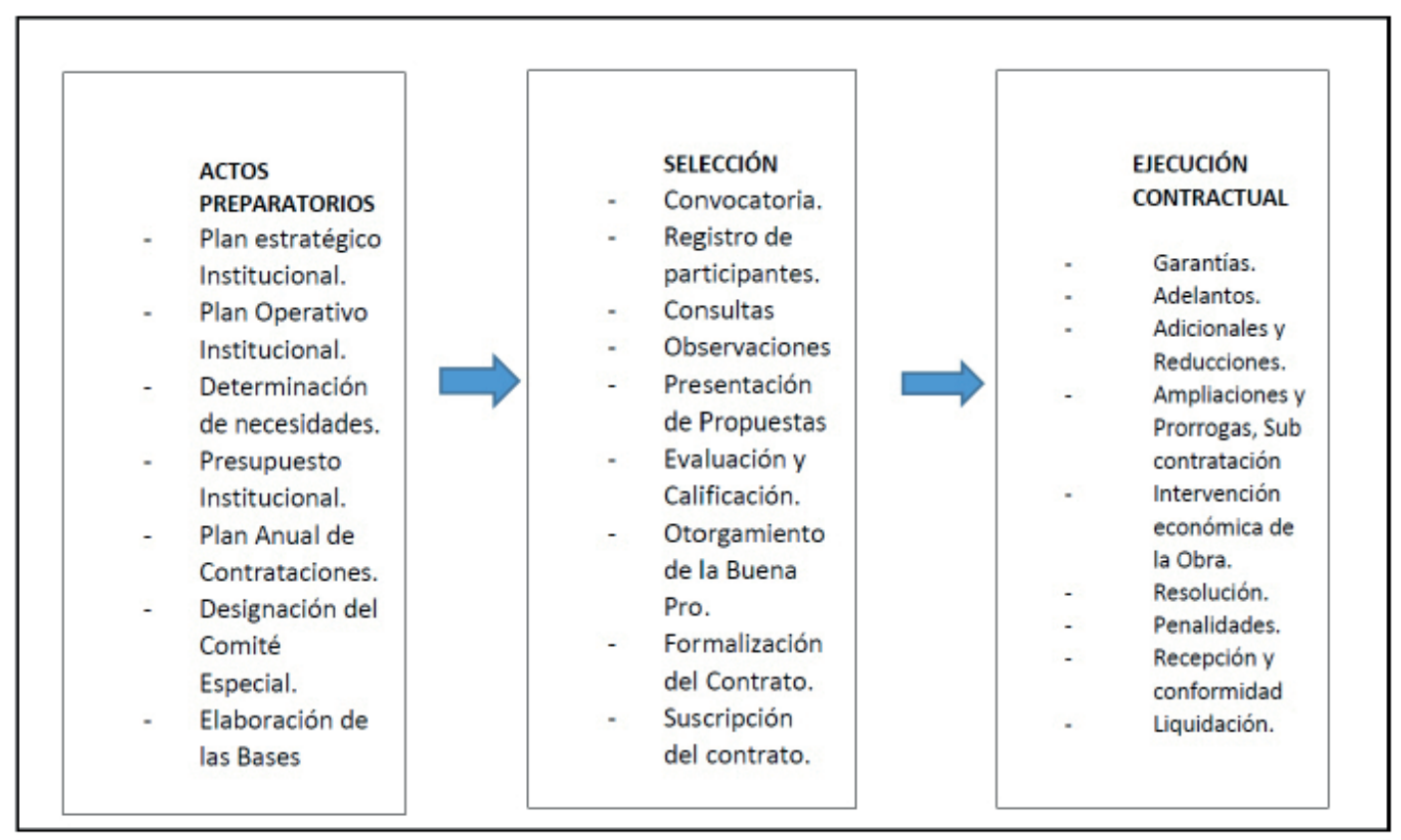

Figura 1. Fases de la contratación estatal Fuente: Hernández (2012)

La etapa de los actos preparatorios incluye todos los procedimientos ejecutados por la entidad desde la planificación de las obras a ejecutar en la que se contempla la solicitud de presupuesto para la ejecución de la obra, la formulación del expediente técnico, se determina el valor referencial, se aprueba el expediente de contratación, se designan los miembros del comité, se elaboran y aprueban las bases adecuadas que conformarán las reglas de juego a las que se someterán participantes y entidad y que han sido programadas por el Comité Especial. Incluyen el calendario del proceso, las exigencias mínimas para postularse.

Es en esta etapa en donde se inician los actos de corrupción, de acuerdo a la experiencia como miembro de diversos comités y personal adjunto a estas dependencias.

La gestión para la ejecución de una obra pertenece a un proyecto de inversión pública que debe ser aprobado por el Ministerio de
Economía y Finanzas a través del Invierte.pe, quien se asegurará de la viabilidad del proyecto, el objetivo público y el reintegro de la inversión.

Cuando se ha aceptado, este se convierte en una necesidad que se forja por medio de un requerimiento que debe ser valorado por la entidad con la finalidad de llegar a su ejecución.

En resumen, se detalla el proceso de selección para el otorgamiento de la buena pro a una determinada empresa para la ejecución de una obra pública, tal como lo hemos presentado, los actos de corrupción comienzan desde el inicio del proceso, pues ya de antemano se han determinado en muchas ocasiones ofrecimientos a los miembros del comité quienes además ya habrían ajustado las bases para que solo la empresa favorecida pueda siquiera postular.

Otro dato si se quiere curioso es que estos actos de corrupción muchas veces se 
convierten en un secreto a voces, donde inclusive todos los miembros de la entidad conocen el hecho, pero no se toman medidas para evitarlo, ni para castigar a los culpables.

Esta situación ha llegado a límites tan exorbitantes que ha involucrado la reputación de los cuatro últimos gobiernos nacionales.

Sonados y comentados casos de corrupción han sido de dominio público. Incluso presidentes y ex presidentes han sido salpicados por los escándalos provocados por la corrupción.

Algunos insisten en declarar su inocencia y auto catalogarse como "perseguidos políticos" como es el caso de Alan García, acusado por principalmente sobre la adjudicación de la línea 1 del metro de Lima (tramos 1 y 2) al consorcio encabezado por Odebrecht.

A pesar de que la empresa Odebrecht ha admitido el pago de 8 millones de dólares en sobornos a funcionarios del segundo gobierno aprista por esta adjudicación, García ha solicitado asilo político en la embajada uruguaya, el cual ha sido denegado.

Otro exmandatario se vio envuelto en esta serie de hechos que involucran a la empresa Odebrecht, el expresidente Pedro Pablo Kuzcysnki (PPK). Ya que en diciembre, esta constructora, protagonista en numerosos escándalos de corrupción en varios países de América Latina, informó al Congreso peruano que había pagado 4,8 millones de dólares a dos firmas de asesoría vinculadas a Kuczysnki.

Textualmente, la empresa señala que realizaron un pago de 782,207 dólares a Westfield Capital, empresa de asesoría de banca de inversión radicada en Miami, Estados Unidos, y perteneciente al presidente del Perú.
Algunos de los pagos sucedieron cuando PPK era funcionario durante el gobierno del expresidente Alejandro Toledo, cuyo periodo presidencial ocurrió entre 2001 a 2006.

Ante la información, el Congreso Nacional solicitó la renuncia de PPK por su supuesta vinculación con esas empresas. Kuczynski, de 79 años, inicialmente negó haber recibido dinero de Odebrecht. Luego negó haber recibido alguna coima, pero reconoció haber trabajado como asesor.

El gobernante no renunció y en diciembre del pasado año salió airoso del proceso de destitución en el Congreso, al que ya no deberá someterse, pues ha renunciado de manera voluntaria.

A pesar de todo, PPK a diferencia de Alan García no ha intentado escapar y actualmente se encuentra enfrentando un juicio.

Tal como hemos podido ver hasta ahora, en el país, se observa un panorama en el que pareciera que no existe algún funcionario de alto cargo y con acceso al patrimonio público que tenga las manos limpias.

Esto representa un verdadero caos, pues el Estado solicita bienes y/o servicios, por medio de funcionarios encargados de contratarlos o comprarlos siguiendo los ordenamientos fundados para avalar principios públicos como la ética, la probidad y la economía en la selección de las ofertas, o de otro modo, eligen no tomar en cuenta estos principios contratando a quien ofrezca el pago de un soborno.

El delegado tiene el poder de, por ejemplo, restringir los proponentes, y garantizar una selección determinada, delimitando y aprobando variables de conveniencia para un contratista en particular no en beneficio del estado. 
Para castigar estos delitos de corrupción, el Estado Peruano ha impuesto una serie de penas dependiendo del acto cometido.

Dicha clasificación señala que para los procesos de contratación pública, no se ha podido detallar todos los tipos de actos de corrupción y falta de ética del funcionario que ocurren en la contratación de obras, por cuanto dicha disposición discurre como único sujeto del castigo al funcionario público.
Como hemos señalado existen múltiples situaciones que no encajan obligatoriamente en el tipo penal descrito, pero que sí representan delitos contra la administración pública y han sido clasificados penalmente, tales como los delitos de concusión, cobro indebido, cohecho (en sus diversas formas), la negociación incompatible, el tráfico de influencias y el enriquecimiento ilícito, entre otros que se detallan en la tabla 1.

Tabla 1. Penas impuestas por la comisión de actos de corrupción

\begin{tabular}{|l|c|c|l|}
\hline \multicolumn{1}{|c|}{ DELITOS } & $\begin{array}{c}\text { ACTORES } \\
\text { FORMALES }\end{array}$ & $\begin{array}{c}\text { ACTORES } \\
\text { INFORMALES }\end{array}$ & \multicolumn{1}{c|}{ PENAS } \\
\hline Colusión simple agravada & $x$ & & $3-6$ años 6-15 años \\
\hline Concusión & $x$ & & $2-8$ años \\
\hline Cobro indebido & $x$ & & $1-4$ años \\
\hline Cohecho pasivo propio & $x$ & & $5-8$ años \\
\hline Cohecho pasivo impropio & $x$ & & $5-8$ años \\
\hline Cohecho pasivo específico & $x$ & & $365-700$ días \\
\hline Cohecho activo genérico & & $x$ & $4-6$ años \\
\hline Cohecho activo específico & & $x$ & $5-8$ años \\
\hline Negociación incompatible & $x$ & & $4-6$ años \\
\hline Tráfico de influencias & & $x$ & $4-8$ años \\
\hline Enriquecimiento ilícito & $x$ & & $5-10$ años 8-18 años \\
\hline
\end{tabular}

Fuente: Hernández (2015) 
Como puede observarse, en el listado anterior, la mayor parte de los delitos tipificados tiene como sujeto del delito la corrupción y por ende de la sanción al funcionario público, el cual es un actor formal dentro de la contratación pública de obras.

Solo tres delitos contemplan la posibilidad de sancionar a los terceros (actores informales) que, en estricto, son quienes fomentan y solventan la corrupción en el sector público.

Esto nos da una clara muestra de que en temas de delitos de corrupción contra la administración pública los verdaderos actores que la fomentan, y tienen una mayor injerencia en que esta ocurra, también son los que tienen mayor posibilidad de disfrazar su actuación mediante actos no tipificados. (Hernández, 2012).

\section{CONCLUSIONES}

La historia de las contrataciones públicas en el Perú ha estado plagadas de actos corrupción desde pequeñas escalas a los más altos niveles.

Por ello es necesario que los funcionarios encargados de esta labor se adhieran a los principios tipificados en el código de ética de la función pública.

Este contiene diferentes principios, deberes y restricciones éticas, por medio de las cuales se rige el accionar de los funcionarios públicos de las distintas entidades que conforman la administración pública peruana.

Se debe tener en cuentas que los objetivos de la función pública son el servicio al Estado, así como la creación de mayores niveles de eficiencia del aparato estatal y por ende debe darse prioridad al uso de los fondos públicos.
Los procedimientos para la contratación de obras públicas se hallan nítidamente delimitados en las leyes, estatutos y normativas, las cuales señalan específicamente las gestiones a seguir en cada una de las fases del proceso; no obstante, los operarios de la norma se las han arreglado para realizar actos que desvían las normas, bien sea de manera formal o mediante el requerimiento de exigencias innecesarias que confinan la libre participación de postores o la direccionan a un licitador que ha ofrecido la respectiva coima, o a través de formas ilegales especificadas o no en las leyes.

Otra de las conclusiones válidas que podemos resaltar es que los actos de corrupción no solo afectan a la nación en su conjunto, o sea, no sólo a los propios organismos públicos, sino también a las empresas y a la sociedad en su conglomerado.

Por estos motivos, una Política Pública de Prevención y querella contra la corrupción es esencial a nivel nacional.

Los actores que participan en los actos de corrupción tienen diferentes motivaciones, pero, ésta siempre será desfavorecedora para el orden público y el marco legal.

De igual forma, esta participación es imprevisible y aunque exista cierto nivel de concordancia entre los actores, no se puede resumir que sea una organización mancomunada e hilada por cuanto los actores no siempre actúan de la misma forma, ni con los mismos motivos, por ende, su participación deberá examinarse en cada caso particular.

Finalmente, cualquier esfuerzo por contrarrestar los actos de corrupción o subsanar la regulación de los procedimientos 
de contrataciones de obras no solucionará la corrupción mientras que el problema involucre la acción de las personas que tienen en su poder la ejecución de los ordenamientos de contratación de obras, por lo cual los investigadores que intenten resolver el problema deben tener en cuenta la participación de los funcionarios y su ética.

\section{REFERENCIAS BIBLIOGRÁFICAS}

Campos, C. (2007). Contratación Pública y Corrupción. Un análisis particular de los principios rectores de la contratación administrativa. Revista de Ciencias Jurídicas. 112.

Centro de Estudios de Responsabilidad Social, Emprendimiento y Sosteniblidad - CERES, CENTRUM Católica. (2017). Crecimiento sostenido, competitividad y desarrollo económico regional del Perú. Strategia, 40-44.

PCM. (2005). Ley Código de Ética de la Función Pública. Ley Código de Ética de la Función Pública. Lima, Perú.

Gorbaneff, Y., y Cabarcas, G. (2009). Equilibrio Económico y la Contratación Pública en Colombia. Revista de Derecho. 31.

Hernández, S. (2012). Estructura de las redes de corrupción en los procesos de selección de obras públicas en el sector transporte y comunicaciones entre los años 2005 y 2010. Perú: Pontificia Universidad Católica del Perú, Maestría en ciencia política con mención gerencia pública.

Huerta, E. (2016). ¿Cómo funciona el cerebro de los corruptos? Esto dice la ciencia. Diario El Comercio. 31.10.2018. Recuperado de: https://elcomercio.pe/tecnologia/ciencias/ funciona-cerebro-corruptos-esto-diceciencia-noticia-506186.

Morales, M. (2009). Corrupción y democracia.
América Latina en perspectiva comparada Santiago de Chile. En: Gestión y Política Pública. Santiago de Chile: Universidad Diego Portales.

Mujica, J. (2011). Micropolíticas de la Corrupción. Redes de poder y corrupción en el Palacio de Justicia. Lima: Asamblea Nacional de Rectores. Instituto de Estudios Universitarios.

Peña, S. (2005). Psicoanálisis de la Corrupción. Lima: Peisa.

Rose-Ackerman, S. (2010). La corrupción y los gobiernos: causas, consecuencias y reforma. Madrid: Madrid: Siglo Veintiuno.

Zegarra, L. (2002). Causas y consecuencias económicas de la corrupción: un análisis teórico y empírico . Lima: Centro de Investigaciones de la Universidad del Pacífico. 